Original Paper http://ajol.info/index.php/ijbcs http://indexmedicus.afro.who.int

\title{
Influence du génotype et du nombre de feuilles sur la croissance en pépinière des boutures du colatier (Cola nitida [Vent.] Schott et Endlicher.)
}

\author{
Drolet Jean-Marc SERY ${ }^{1 *}$, Bouadou BONSSON ${ }^{1}$, Rachel GNOGBO $^{2}$, Nadré GBEDIE ${ }^{1}$, \\ Yaya OUATTARA ${ }^{1}$, Hyacinthe LEGNATE ${ }^{1}$ et Zagbahi Jules KELI ${ }^{1}$ \\ ${ }^{I}$ Centre National de Recherche Agronomique (CNRA), Station de Recherche de Man, \\ B.P. $440 \mathrm{Man} /$ Côte d'Ivoire. \\ ${ }^{2}$ Université Lorougnon Guédé, Daloa, Côte d'Ivoire. \\ *Auteur correspondant ; E-mail: sery.jeanmarc@yahoo.fr, Tel: (+225) 08946103
}

\section{RESUME}

Malgré son importance socioéconomique, la production ivoirienne de noix de cola est confrontée à plusieurs difficultés. Le manque de techniques culturales éprouvées constitue une des contraintes pour la domestication et la culture du colatier. Il est donc nécessaire de mettre au point des techniques adaptées à l'espèce cultivée. L'objectif de cette étude est d'optimiser la méthode de bouturage sous tunnel du colatier (Cola nitida), face aux inconvénients de la multiplication générative. Le dispositif expérimental utilisé est un Split-Splot avec deux facteurs : le génotype avec cinq modalités (5 génotypes) et le nombre de feuilles avec cinq (05) modalités (1, 2, 3, 4 et 5 feuilles diminuées de moitié). Nous avons le génotype en grande parcelle et le nombre de feuilles en petite parcelle. L'aptitude des génotypes au bouturage a été évaluée à six mois à travers le taux de survie et la mesure des paramètres de croissance et de développement. Le génotype L18A1D9 a montré la meilleure aptitude au bouturage avec un taux de survie de 41,7\%. Les boutures avec 4 feuilles diminuées de moitié ont eu le meilleur taux de survie $(41,7 \%)$, un bon enracinement des boutures vivantes $(88,9 \%)$, une biomasse totale fraîche $(4,2 \mathrm{~g})$ et totale sèche $(1,7 \mathrm{~g})$ élevée. Le succès du bouturage sous tunnel du colatier est tributaire du génotype et du nombre de feuilles. Le nombre de feuilles recommandé par bouture est de quatre (4) pour le bouturage du colatier sous tunnel.

(C) 2019 International Formulae Group. All rights reserved.

Mot clés : Bouturage, nombre de feuilles, Cola nitida, génotype.

\section{Influence of genotype and number of leaves on the growth in nurseries of cola cuttings (Cola nitida [Wind.] Schott and Endlicher.)}

\begin{abstract}
Despite its socio-economic importance, kola nut production faces several challenges. Domestication and cultivation of kola requires the adoption of propagation techniques adapted to the crop species. The objective of this study is to optimize kola (Cola nitida) vegetative propagation by cutting under tunnel. The experimental design used was a split-splot with two factors: genotype with five modalities (5 genotypes,) and leave number with five (05) modalities (1, 2, 3, 4 and 5 leaves cut in half). The ability of genotype to cutting propagation was assessed by measuring growth parameters. The survival rate of the cuttings was evaluated at
\end{abstract}


six months. Root growth, number of new leaves, height gain and biomass were subsequently measured. Genotype L18A1D9 has the best ability for vegetative propagation by cuttings with a survival rate of $41.7 \%$. To optimize vegetative propagation by cuttings under tunnel, 4 leaves cut in half by cuttings must be used for acceptable success rate (41.7\%), good rooting (88.9\%), a high total fresh biomass ( $4.2 \mathrm{~g}$ ) and total biomass (1.7 g). The success of kola vegetative propagation depends of genotype and the leave number per cutting. The number of leaves per cuttings recommended per cuttings is four (4) for kola vegetative propagation.

(C) 2019 International Formulae Group. All rights reserved.

Keywords: Cuttings, number of leaves, Cola nitida, genotype.

\section{INTRODUCTION}

Le colatier (Cola nitida [Vent.] Schott et Endlicher.) appartient à la famille des Malvaceae (Whitlock et al., 2001). Le genre Cola, endémique en Afrique renferme environ 140 espèces (Adenuga et al., 2012) dont une cinquantaine est originaire d'Afrique de l'Ouest (Adebola, 2003).

Le colatier pousse dans les régions d'Afrique tropicale et équatoriale où il peut atteindre plus de 25 mètres (Ouattara et al., 2018). Il produit la noix de cola qui est très prisée en Afrique et dans le monde, pour ses usages socioculturels (mariages, baptêmes, funérailles) et industriels (savon, teinture, médicaments et boissons gazeuses) (Asogwa et al., 2006 ; Biego et al., 2009). La noix de cola fait l'objet d'un important réseau d'échanges commerciaux entre les pays africains et entre l'Afrique et le reste du monde.

L'espèce cultivée en Côte d'Ivoire est Cola nitida [Vent.] Schott et Endlicher. Le pays est le premier producteur mondial de noix de cola devant le Nigéria, avec une production estimée à 260.000 tonnes/an de noix fraîches (MINADER, 2018), pour un chiffre d'affaire d'environ 100 milliards de francs CFA (Aloko-N'Guessan, 2000). Malgré son importance socioéconomique, la production de noix de cola est confrontée à plusieurs difficultés (Berté, 2009). L'expansion de cette culture est confrontée à l'absence de matériel végétal performant, la germination lente et échelonnée de la noix (Mbete et al., 2011) et les difficultés des producteurs pour accéder à une assistance technique (Berté, 2009).

Le faible taux d'expansion constaté est en partie attribué à la dormance prolongée des semences, à la germination irrégulière des graines et à la faible performance de la méthode de semis direct pratiquée par les paysans (Gbédié et al., 2017). L'expansion de la culture est fortement tributaire des arbres sylvestres qui subissent la déforestation. La domestication du colatier suppose l'adoption de techniques de multiplication adaptée à l'espèce cultivée (Tchoundjeu et al., 2002a).

La multiplication générative du colatier via l'usage des noix semences, aboutit à la dissociation des caractères de la plante mère au niveau de sa descendance. Cette situation s'explique par l'allogamie du colatier (Odutayo et al., 2018).

La multiplication végétative (bouturage, marcottage et greffage) apparait comme une alternative durable (Degrande et Facheux, 2002 ; Paluku et al., 2018b) pour la culture du colatier. En effet, un jeune plant obtenu par multiplication végétative d'un arbre déjà adulte adopte d'emblée le mode de croissance sympodiale. De sorte que, deux à trois ans après la plantation, un tel plant entre en production. La multiplication végétative permet donc de raccourcir le cycle du colatier et d'obtenir des populations homogènes d'arbres dans les plantations.

Dès le début des travaux d'amélioration de la production du colatier au Nigéria, en Côte d'Ivoire et en République Centrafricaine, les recherches effectuées ont montré qu'un nombre élevé de facteurs influencent le taux de survie et l'enracinement des boutures. Ces facteurs sont, entre autres, le génotype, le nombre de feuilles, le type de substrat, la longueur des boutures, le type de rameau, la concentration en phytohormones, le nombre et la position de nœuds (Paluku et al., 2018a). En outre, les taux de survie et d'enracinement 
obtenus ont été relativement faibles, inférieurs à $15 \%$. Il convient donc de continuer les travaux pour améliorer ces taux.

Notre étude entre dans ce cadre. Elle se propose d'évaluer l'influence de deux facteurs: le génotype du matériel végétal utilisé et le nombre de feuilles par rameau au niveau de la méthode de multiplication végétative par bouturage.

\section{MATERIEL ET METHODES}

\section{Présentation du site d'expérimentation}

L'essai a été mis en place en novembre 2018, au début de la saison sèche, sur le site de la pépinière du Centre National de Recherche Agronomique (CNRA) de Man, à $l^{\prime}$ Ouest de la Côte d'Ivoire $\left(7^{\circ} 19.130\right.$ ' $\mathrm{N} ; 8^{\circ}$ $\left.19.452^{\prime} \mathrm{W}\right)$. La pluviométrie de la zone de Man est de type monomodal. La saison sèche s'étend généralement d'octobre à mars et la saison des pluies d'avril à septembre. Le site a reçu une pluviométrie annuelle moyenne en 2018 de $1632 \mathrm{~mm}$. La température en 2018 a varié de $23{ }^{\circ} \mathrm{C}$ à $27^{\circ} \mathrm{C}$.

\section{Matériel végétal}

Le matériel végétal utilisé était composé de 1500 boutures de colatier. Ces boutures ont été prélevées sur cinq (5) génotypes de la parcelle D9, plantée en 2009, dans la station CNRA de Divo. Ces cinq génotypes sont identifiés par les codes suivants : L6A1D9 ; L10A1D9 ; L16A1D9 ; L18A1D9 et L22A1D9. Ces génotypes ont été choisis sur la base de leur productivité.

\section{Matériel technique}

Le matériel technique utilisé pour cette étude est constitué de sécateurs pour le prélèvement et l'habillage des boutures et d'un décamètre pour les mesures de circonférences et de hauteurs des boutures. Des sachets plastiques de couleur blanche, fermés hermétiquement à l'aide d'une agrafeuse et conservés dans des glaciaires ont été utilisés pour la conservation des boutures lors du transport. Un substrat à base de terre humifère a été collecté pour le remplissage des pots (sachet de $30 \mathrm{~cm}$ hauteur $/ 15 \mathrm{~cm}$ diamètre). Le fongicide IVORY* $80 \% \mathrm{WP}$ (m.a: Manèbe, Fabricant: ARYSTA Life science) a été utilisé pour le traitement préventif dès le repiquage des boutures.

\section{Description de l'essai}

Dispositif expérimental

Le dispositif expérimental utilisé est un Split-Splot avec deux (2) facteurs que sont : le génotype et le nombre de feuilles par bouture. Le génotype constitue le facteur principal avec cinq (05) modalités: G1 : L5A1D9 ; G2 : L10A1D9 ; G3 : L16A1D9 ; G4 : L18A1D9 et G5 : L22A1D9. Le nombre de feuilles par bouture constitue le facteur secondaire avec cinq (05) modalités : F1: 1 feuille par bouture, $\mathrm{F} 2: 2$ feuilles par bouture, F3 : 3 feuilles par bouture, F4: 4 feuilles par bouture et F5: 5 feuilles par bouture. Les feuilles ont été diminuées toutes de moitié. Vingt pots (sachets), contenant chacun une bouture du même génotype, ont été utilisés par traitement. Les traitements ont été répétés trois (03) fois. Au total, 300 boutures provenant du même génotype ont été utilisées pour cet essai.

La méthode de bouturage utilisé a été le bouturage sous tunnel. L'armature du tunnel devant abriter les pots (sachets) contenant les boutures était faite d'arceaux de 2,4 m relié par des lattes de bambou. Le tunnel était recouvert d'une bâche plastique transparente d'épaisseur 100 microns et de largeur 2,6 m plaquée au sol sur les côtés et aux extrémités au moyen de pierres et de bambous. Les tunnels ont été disposés sous un abri de pépinière qui est constitué d'une charpente de palmes de $2 \mathrm{~m}$ de hauteur qui laisse passer environ $50 \%$ de la lumière totale.

\section{Prélèvement et repiquage des boutures}

Trois cents boutures par génotype ont été prélevées, tôt le matin, sur les rameaux terminaux semi-lignifiés dans une parcelle de la station de Divo. Un total de 1500 boutures a été ainsi prélevé à l'aide de sécateur pour les 5 génotypes étudiés. Les boutures de chaque arbre ont été transportées dans des sachets plastiques contenant de l'eau afin de réduire au maximum leur déshydratation. Les sachets fermés à l'aide d'agrafeuse, ont été mis dans 
des glaciaires afin de maintenir l'humidité. La taille des boutures était de $12 \mathrm{~cm}$.

Pour la mise en place de cet essai, dans chaque tunnel, les pots ont été remplis de substrat (terre noire) préalablement homogénéisé et désinfecté. Les boutures ont été repiquées à la main à une profondeur d'environ $3 \mathrm{~cm}$. Les caractéristiques chimiques du substrat utilisé dans les pots ont été déterminées.

\section{Analyse physico-chimique du substrat}

Le substrat utilisé était le terreau. Ce terreau a été collecté et envoyé à l'état brut au Laboratoire de Pédologie de l'Institut National Polytechnique-Félix HouphouëtBoigny (INPHB) pour analyses. Des paramètres physiques et chimiques ont été déterminés (Tableau 1). Les normes d'interprétation utilisées sont pour le $\mathrm{pH}$ le référentiel pédagogique de l'INRA (INRA, 1995). Les normes d'interprétation de la capacité d'échange cationique (CEC), du phosphore assimilable, de l'azote et de la matière organique sont celles définies par Calvet et Villemin (1986).

\section{Conduite de l'essai}

La disposition des pots et le traitement phytosanitaire ont été effectués la veille de la mise sous tunnels des boutures. Concernant le traitement phytosanitaire, le fongicide IVORY 80\% WP (m.a. manèbe, Fabricant: ARYSTA Life science) a été utilisé (70 g dans 2 litres d'eau appliqués dans les pots). Les boutures ont été arrosées chaque 2 jour.
Pour la mesure des paramètres de croissance, sur les 20 pots de chaque traitement, le nombre de plants vivants a été noté à six mois afin de quantifier le taux de survie. Le développement racinaire, le nombre de nouvelles feuilles, le gain en hauteur de la plantule et la biomasse (fraîche et sèche) ont été évalués au bout de six mois. La biomasse sèche a été évaluée à l'aide d'une balance électronique après séchage à l'air libre pendant deux semaines.

\section{Analyses statistiques}

Pour les paramètres examinés, une comparaison des moyennes entre les différents facteurs et les différents traitements a été faite à travers l'analyse de la variance simple (ANOVA) ou multi-variée (MANOVA). Lorsqu'une différence significative est observée entre les traitements pour un facteur donné, l'ANOVA ou la MANOVA est complétée par des tests post-hoc en effectuant le test de Newman-Keuls pour identifier les différences significatives entre les moyennes au seuil de $5 \%$. Pour tous ces tests, le logiciel STATISTICA 7.1 a été utilisé. Le taux de survie (Ts) et le gain de croissance en hauteur (Gh) ont été calculés selon les formules suivantes :

$$
\begin{gathered}
\text { Ts }=\left(\frac{\text { Nombre de plants vivants }}{\text { Nombre initial de plants }}\right) * 100 \\
\text { Gh }=\left(\frac{\text { Hauteur finale }- \text { Hauteur initiale }}{\text { Hauteur initiale }}\right) * 100
\end{gathered}
$$

\begin{tabular}{|c|c|c|c|c|c|}
\hline $\begin{array}{l}\text { Caractéristiques } \\
\text { physico-chimiques } \\
\text { du terreau }\end{array}$ & $\mathbf{p H}_{\text {eau }}$ & C & M.O & $\mathbf{N}$ & $\begin{array}{l}\text { P. ass } \\
(\mathrm{mg} / \mathrm{kg})\end{array}$ \\
\hline & 6,7 & 1,99 & 3,43 & 0,17 & 38 \\
\hline & $\begin{array}{l}\text { CEC } \\
\text { (cmol.kg-1) }\end{array}$ & $\begin{array}{l}\mathrm{Ca}^{2+} \\
(\mathrm{cmol} \cdot \mathrm{kg}-1)\end{array}$ & $\begin{array}{l}\mathrm{Mg}^{2+} \\
\text { (cmol.kg-1) }\end{array}$ & $\begin{array}{l}\mathrm{K}^{+}(\mathrm{cmol} \cdot \mathrm{kg}- \\
1)\end{array}$ & $\begin{array}{l}\mathrm{Na}^{+} \\
(\mathrm{cmol} \cdot \mathrm{kg}-1)\end{array}$ \\
\hline & 11,84 & 3,433 & 0,789 & 0,157 & 0,072 \\
\hline
\end{tabular}

Tableau 1 : Caractéristiques physico-chimiques du terreau.

$\mathrm{pH}_{\text {eau }}$ : potentiel Hydrogène, C : Carbone, M.O : Matière organique, N: Azote, P. ass : phosphore assimilable. 


\section{RESULTATS}

Caractérisation physico-chimique du substrat de culture

Le substrat utilisé a un $\mathrm{pH}$ proche de 7 (Tableau 1). Il a une teneur moyenne en matière organique $(\mathrm{M}=3,43 ; \mathrm{C}=1,99$; $\mathrm{N}=0,17$ ) et est pauvre en phosphore assimilable (P. ass $=38 \mathrm{mg} / \mathrm{kg})$. L'estimation de la capacité d'échange cationique (C.E.C $=11,84)$ révèle un substrat avec une capacité de stockage des éléments nutritifs $\left(\mathrm{Ca}^{2+}, \mathrm{Mg}^{2+}, \mathrm{K}+, \mathrm{Na}^{+}\right)$acceptable.

\section{Influence du génotype et du nombre de feuilles sur le taux de survie des boutures}

Les taux de survie des boutures en pépinière ont été évalués à six mois. Le taux de survie moyen général à six mois est faible $(5,9 \%)$. Ce taux de survie varie selon le nombre de feuilles et les génotypes. Une analyse factorielle de variance a été faite (Tableau 2) afin de déterminer l'influence respective des deux (2) facteurs étudiés : Nombre de feuilles et Génotype et leur Interaction sur la variation du taux de survie constatée. Cette étude a montré que le Nombre de feuilles laissé par bouture et le Génotype du colatier ont un impact significatif sur le taux de survie des boutures en pépinière. Elle a montré qu'il n'existe aucune interaction entre le Nombre de feuilles et le Génotype $(p=0,066336)$. Le nombre de feuilles à laisser par bouture n'est donc pas fonction du génotype.

L'analyse des données collectées à six mois (Figure 1) révèle que les boutures avec 4 feuilles (Figure 1) ont le taux de survie moyen le plus élevé $(13,3 \%)$ quel que soit le génotype.

La comparaison des taux moyens de survie a permis d'identifier 3 génotypes (Figure 2). Le génotype L18A1D9 avec le taux moyen de survie le plus élevé (20\%), est le plus apte au bouturage. Les génotypes
L10A1D9, L22A1D9, L5A1A9 ont montré une très faible aptitude au bouturage respectivement $0,3 \%, \quad 0 \%$ et $1,3 \%$. Le génotype L16A1D9 avec un taux moyen de survie de $8 \%$, a des caractéristiques intermédiaires entre ces deux précédents groupes de génotypes.

Après six mois de culture en serre, le meilleur taux de survie de $41,7 \%$ a été obtenu avec le génotype L18A1D9 pour 4 feuilles laissées par bouture (Figure 3).

Influence du génotype et du nombre de feuilles sur la croissance et le développement du colatier.

Les données de gain en hauteur, le nombre de nouvelles feuilles et la biomasse collectée ont été analysées.

Influence du génotype et du nombre de feuilles par bouture sur le développement racinaire

L'analyse des données de développement racinaire (boutures racinées, nombre de racines, longueur de la racine principale) a révélé que le génotype $(p=0,000)$, le nombre de feuilles $(p=0,000)$ et l'interaction génotype-nombre de feuilles $(p=0,009)$ ont un effet significatif sur la croissance et le développement racinaire des plants de colatiers lors du bouturage sous tunnel en pépinière. Les résultats de l'ANOVA multi varié sont consignés dans le Tableau 3 .

Les résultats présentés dans le Tableau 4 montrent que les meilleurs taux d'enracinement ont été obtenus avec les génotypes L18A1D9 et L16A1D9. Les taux d'enracinement les plus élevés pour le génotype L18A1D9 ont été obtenus pour les boutures avec 4 feuilles (88,9\%), 5 feuilles $(77,8 \%)$ et 3 feuilles $(66,7 \%)$. Tandis que pour le génotype L16A1D9, le meilleur taux a été obtenu pour les boutures de 5 feuilles $(77,8 \%)$. Le nombre de racines est plus important mais pas statiquement différent des 
autres (Tableau 4) pour le génotype L18A1D9 avec les boutures de 5 feuilles $(2,3)$ et 4 feuilles $(1,4)$. Cependant, les racines formées sont significativement plus longues pour les boutures avec 4 et 5 feuilles $(10,2$ et 8,0 respectivement). Il est suivi du génotype L16A1D9 avec un nombre significatif de racines ( 5 racines) pour les boutures avec 5 feuilles et 0,3 racine pour les boutures de 4 feuilles.

\section{Influence du génotype et du nombre de feuilles sur le gain en hauteur et la formation de nouvelles feuilles}

Les gains moyens en hauteur (Tableau 5) ont été notés essentiellement avec le génotype L18A1D9 avec un gain en hauteur élevé de 15,8\% pour les boutures avec 5 feuilles et $13,15 \%$ pour les boutures avec 4 feuilles.

Il faut ajouter également que ce génotype a produit en moyenne plus de nouvelles feuilles (Tableau 6), 4, 3 et 2 feuilles pour les boutures avec 5,4 et 2 feuilles respectivement.

Outre ce génotype, le génotype L16 A1D9 a également produit de nouvelles feuilles 0,8 et 1,1 respectivement pour les boutures de 5 et 4 feuilles.
Influence du génotype et du nombre de feuilles sur la biomasse des plants de colatiers en pépinière

L'étude des facteurs responsables de la variation de la biomasse des plants de colatiers (Tableau 5) a montré l'effet significatif des 2 facteurs étudiés. L'analyse détaillée des données sur la matière fraîche totale et la matière sèche totale (Tableau 6) a mis en évidence des teneurs en matière fraîche totale élevées pour les génotypes L18A1D9 et L16A1D9. Pour le génotype L18A1D9, elles sont de 4,2 $\mathrm{g}, 3,58 \mathrm{~g}$ et $2,9 \mathrm{~g}$ avec $4,5,3$ feuilles respectivement. Pour le génotype L16A1D9, elle est de 2,98 g, avec 5 feuilles. Pour la matière sèche, le constat est le même pour L18A1D9 avec des teneurs en matières sèches élevées et statistiquement différentes pour les boutures à 5,4 et 3 feuilles.

Comme nous l'avons montré dans le Tableau 6, la MANOVA a mis en évidence l'effet significatif du génotype et du nombre de feuilles sur la biomasse en générale. Les génotypes L18A1D9 $(1,1 \mathrm{~g})$ et L16 A1D9 (0,5 g) ont la matière sèche aérienne la plus importante (Figure 4 a). Il en est de même pour la matière sèche racinaire (Figure $4 \mathbf{b}$ ). Pour le nombre de feuilles (Figure $4 \mathbf{c} ; \mathbf{d}$ ), les plus grandes teneurs en matières sèches aériennes et racinaires sont obtenues pour les boutures ayant 4 et 5 feuilles.

Tableau 2 : Analyse de Variance (ANOVA) des facteurs « Nombre de feuilles » et « Génotype » sur le taux de survie.

\begin{tabular}{lccccc}
\hline & SC & D.L & MC & F & p \\
\hline Nombre de feuilles & 44,8533 & 4 & 11,2133 & 6,13869 & $\mathbf{0 , 0 0 0 4 2 4}$ \\
Génotype & 173,9200 & 4 & 43,4800 & 23,80292 & $\mathbf{0 , 0 0 0 0 0 0}$ \\
Nombre de feuilles*Génotype & 51,2800 & 16 & 3,2050 & 1,75456 & 0,066336 \\
Erreur & 91,3333 & 50 & 1,8267 & & \\
\hline
\end{tabular}

SC : Somme des carrés ; D.L : Degré de liberté ; MC : Moyenne des carrés ; F : Fischer ; p : Probabilité. 


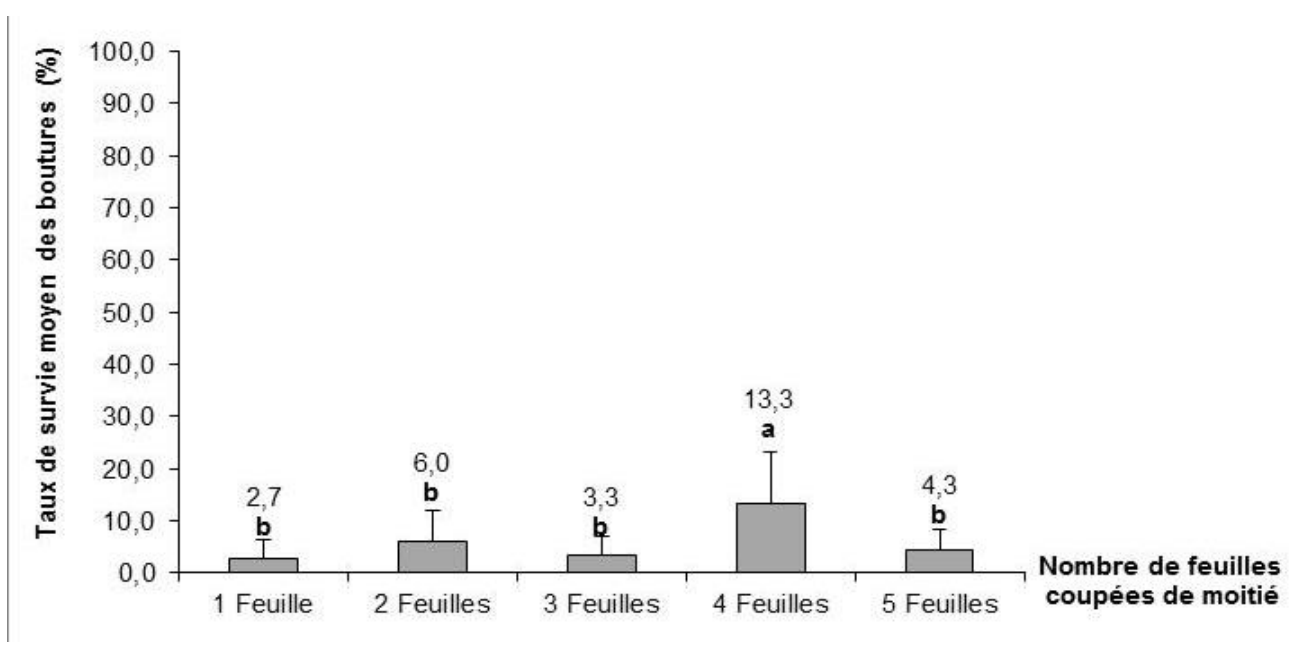

*Les données portant les mêmes lettres ne sont pas significativement différentes au seuil de 5\%. Test de Newman-Keuls.

Figure 1 : Influence du nombre de feuilles par bouture.

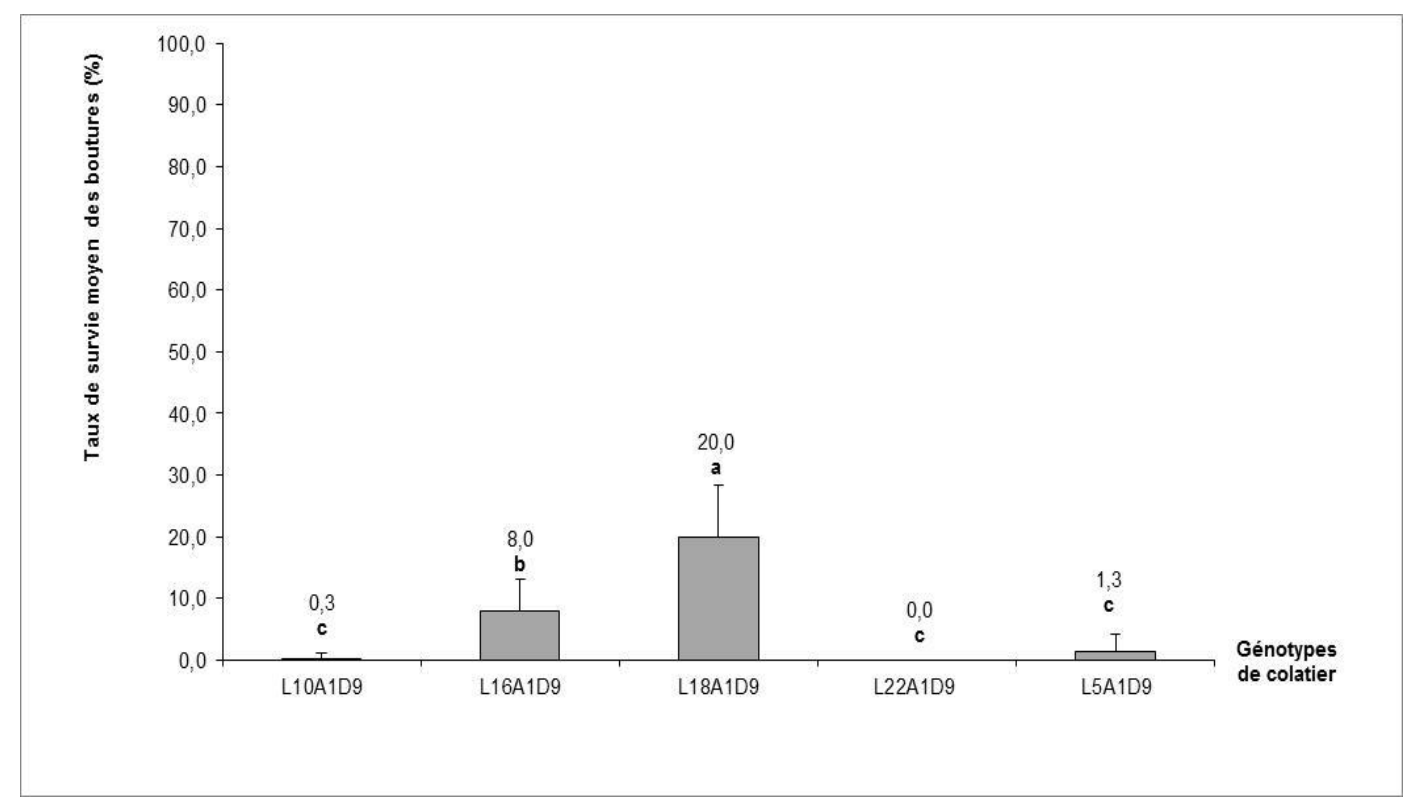

Les données portant les mêmes lettres ne sont pas significativement différentes au seuil de 5\%. Test de Newman-Keuls.

Figure 2 : Aptitudes au bouturage des génotypes testés. 


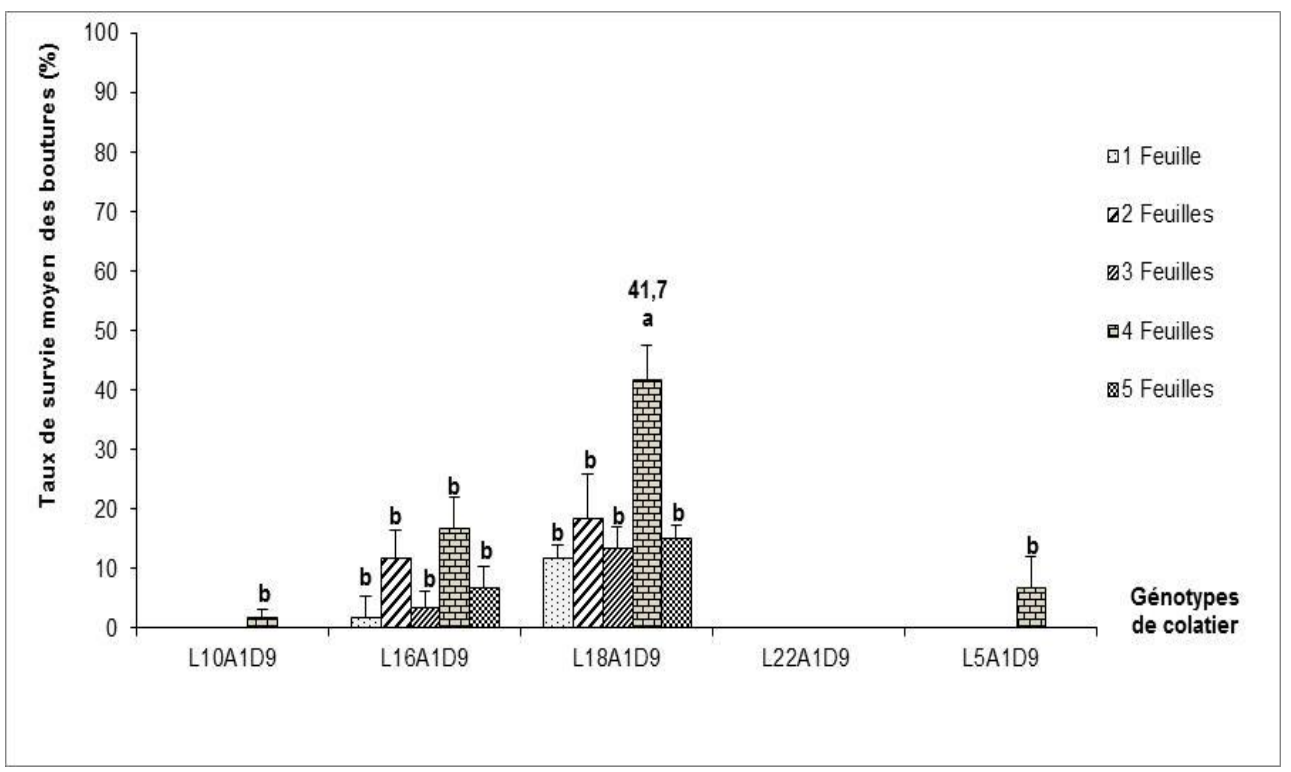

Les données portant les mêmes lettres ne sont pas significativement différentes au seuil de 5\%. Test de Newman-Keuls.

Figure 3: Meilleurs taux de survie des génotypes en fonction du nombre de feuilles à six mois.

Tableau 3: MANOVA factorielle des données de croissance racinaire.

\begin{tabular}{ccc}
\hline & $\mathbf{F}^{*}$ & $\mathbf{P}^{*}$ \\
\hline Génotype & 6,18907 & $\mathbf{0 , 0 0 0 0 0 0}$ \\
Traitement & 3,25176 & $\mathbf{0 , 0 0 0 0 8 1}$ \\
Génotype*Traitement & 1,58776 & $\mathbf{0 , 0 0 9 0 4 5}$ \\
\hline
\end{tabular}

*F : Fischer ; *p : Probabilité.

Tableau 4 : Analyse des données sur les boutures racinées, le nombre et la longueur des racines.

\begin{tabular}{lccccc}
\hline Génotypes & \multicolumn{5}{c}{ Bouture racinée (\%) } \\
\cline { 2 - 6 } & F1 & F2 & F3 & F4 & F5 \\
\hline L10A1D9 & $0 \pm 0^{\mathrm{e}}$ & $0 \pm 0^{\mathrm{e}}$ & $0 \pm 0^{\mathrm{e}}$ & $11,1 \pm 15,7^{\mathrm{e}}$ & $0 \pm 0^{\mathrm{e}}$ \\
L16A1D9 & $0 \pm 0^{\mathrm{e}}$ & $11,1 \pm 15,7^{\mathrm{e}}$ & $11,1 \pm 15,7^{\mathrm{e}}$ & $22,2 \pm 15,7^{\mathrm{de}}$ & $77,8 \pm 32,1^{\mathrm{abc}}$ \\
L18A1D9 & $33,3 \pm 27,2^{\mathrm{bcde}}$ & $33,3 \pm 27,2^{\mathrm{bde}}$ & $66,7 \pm 0,0^{\mathrm{abcd}}$ & $88,9 \pm 15,7^{\mathrm{a}}$ & $77,8 \pm 32,1^{\mathrm{abc}}$ \\
L22A1D9 & $0 \pm 0^{\mathrm{e}}$ & $0 \pm 0^{\mathrm{e}}$ & $0 \pm 0^{\mathrm{e}}$ & $0 \pm 0^{\mathrm{e}}$ & $0 \pm 0^{\mathrm{e}}$ \\
L5A1D9 & $0 \pm 0^{\mathrm{e}}$ & $0 \pm 0^{\mathrm{e}}$ & $0 \pm 0^{\mathrm{e}}$ & $33,3 \pm 47,1^{\mathrm{ce}}$ & $0 \pm 0^{\mathrm{e}}$ \\
\hline & \multicolumn{5}{c}{ Nombres de racines } \\
\hline L10A1D9 & $0,0 \pm 0^{\mathrm{b}}$ & $0,0 \pm 0^{\mathrm{b}}$ & $0,0 \pm 0^{\mathrm{b}}$ & $0,8 \pm 1,1^{\mathrm{b}}$ & $0,0 \pm 0^{\mathrm{b}}$ \\
L16A1D9 & $0,0 \pm 0^{\mathrm{b}}$ & $0,1 \pm 0,1^{\mathrm{b}}$ & $0,1 \pm 0,1^{\mathrm{b}}$ & $0,3 \pm 0,2^{\mathrm{b}}$ & $5,0 \pm 2,8^{\mathrm{a}}$ \\
L18A1D9 & $0,7 \pm 0,5^{\mathrm{b}}$ & $0,7 \pm 0,7^{\mathrm{b}}$ & $1,4 \pm 0,4^{\mathrm{b}}$ & $1,4 \pm 0,3^{\mathrm{b}}$ & $2,3 \pm 1,9^{\mathrm{b}}$ \\
L22A1D9 & $0 \pm 0^{\mathrm{b}}$ & $0 \pm 0^{\mathrm{b}}$ & $0 \pm 0^{\mathrm{b}}$ & $0 \pm 0^{\mathrm{b}}$ & $0 \pm 0^{\mathrm{b}}$ \\
L5A1D9 & $0,0 \pm 0^{\mathrm{b}}$ & $0,0 \pm 0^{\mathrm{b}}$ & $0,0 \pm 0^{\mathrm{b}}$ & $1,1 \pm 1,5^{\mathrm{b}}$ & $0,0 \pm 0^{\mathrm{b}}$ \\
\hline
\end{tabular}




\begin{tabular}{lccccc}
\hline \multicolumn{5}{c}{ Longueur des racines (cm) } \\
\hline & F1 & F2 & F3 & F4 & F5 \\
L10A1D9 & $0,0 \pm 0^{\mathrm{d}}$ & $0,0 \pm 0^{\mathrm{d}}$ & $0,0 \pm 0^{\mathrm{d}}$ & $1,7 \pm 2,4^{\mathrm{d}}$ & $0,0 \pm 0^{\mathrm{d}}$ \\
L16A1D9 & $0,0 \pm 0^{\mathrm{d}}$ & $0,6 \pm 0.8^{\mathrm{d}}$ & $0,5 \pm 0,7^{\mathrm{d}}$ & $1,9 \pm 1,8^{\mathrm{d}}$ & $8,1 \pm 4,5^{\mathrm{abc}}$ \\
L18A1D9 & $3,7 \pm 2,6^{\text {bcd }}$ & $2,3 \pm 2,0^{\text {bd }}$ & $6,3 \pm 0,36^{\mathrm{abcd}}$ & $10,2 \pm 1,6^{\mathrm{a}}$ & $8,0 \pm 3,5^{\mathrm{ac}}$ \\
L22A1D9 & $0 \pm 0^{\mathrm{d}}$ & $0 \pm 0^{\mathrm{d}}$ & $0 \pm 0^{\mathrm{d}}$ & $0 \pm 0^{\mathrm{d}}$ & $0 \pm 0^{\mathrm{d}}$ \\
L5A1D9 & $0,0 \pm 0^{\mathrm{d}}$ & $0,0 \pm 0^{\mathrm{d}}$ & $0,0 \pm 0^{\mathrm{d}}$ & $4,5 \pm 6,36^{\text {bcd }}$ & $0,0 \pm 0^{\mathrm{d}}$ \\
\hline *Sur la même ligne, les données portant les mêmes lettres ne sont pas significativement différentes au seuil de $5 \%$
\end{tabular}

*Sur la même ligne, les données portant les mêmes lettres ne sont pas significativement différentes au seuil de 5\%.

Tableau 5 : MANOVA factorielle des données de la biomasse fraîche et de la biomasse sèche.

\begin{tabular}{clcc}
\hline & Valeur & $\mathbf{F}^{*}$ & $\mathbf{P}^{*}$ \\
\hline Génotype & 0,298006 & 6,02690 & $\mathbf{0 , 0 0 0 0 0 0}$ \\
Traitement & 0,619171 & 2,06322 & $\mathbf{0 , 0 2 4 0 1 0}$ \\
Génotype*Traitement & 0,306351 & 1,43067 & 0,055781 \\
\hline
\end{tabular}

*F : Fischer ; *p : Probabilité.
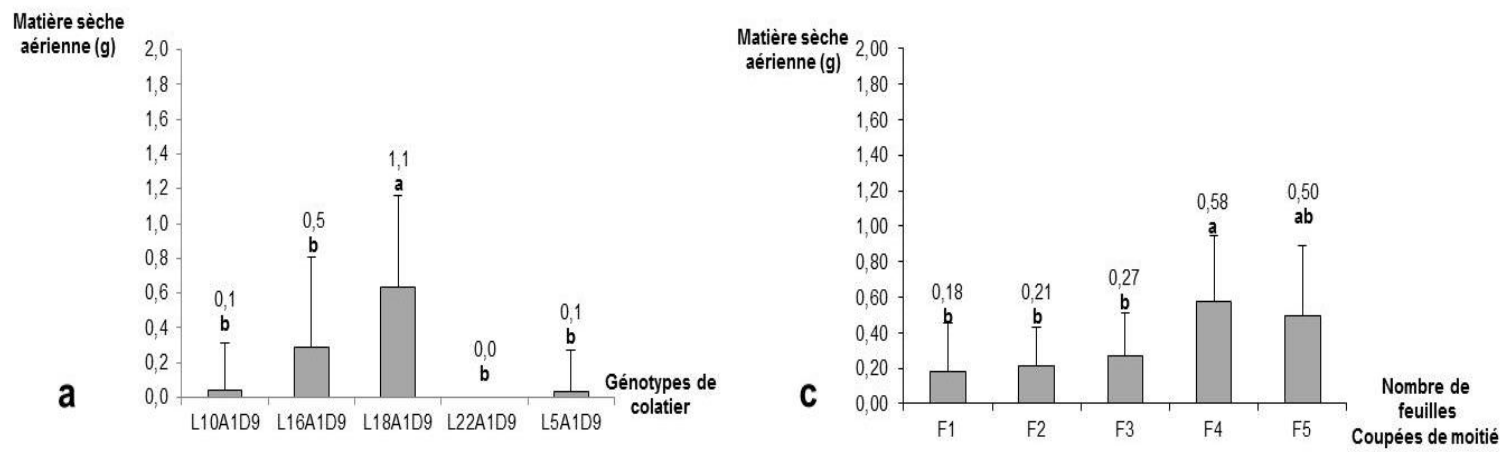

Matière sèche racinaire

(g)
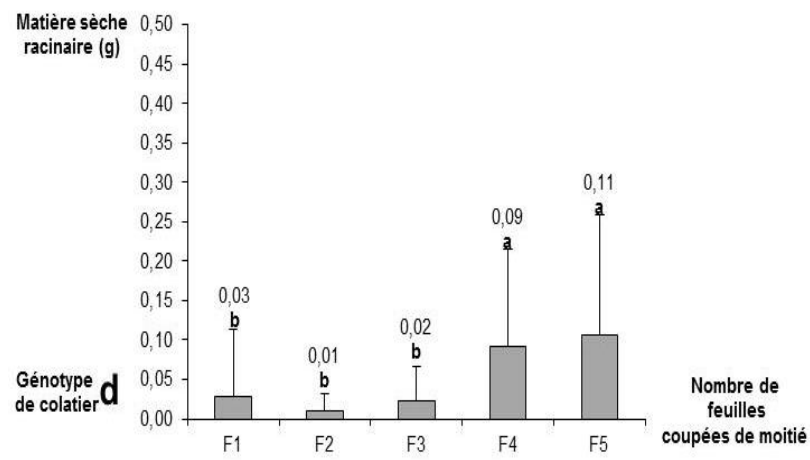

Les données portant les mêmes lettres ne sont pas significativement différentes au seuil de 5\%. Test de Newman-Keuls.

Figure 4: Effet du génotype et du nombre de feuilles sur la matière sèche aérienne et racinaire. 
Tableau 6 : Analyse des données sur le gain en hauteur, le nombre de nouvelles feuilles, la matière fraîche totale et la matière sèche totale.

\begin{tabular}{lccccc}
\hline $\begin{array}{l}\text { Gain en hauteur } \\
(\mathbf{c m})\end{array}$ & $\mathbf{1}$ feuille & $\mathbf{2}$ feuilles & $\mathbf{3}$ feuilles & $\mathbf{4}$ feuilles & $\mathbf{5}$ feuilles \\
\hline L10A1D9 & $0 \pm 0^{\mathrm{bc}}$ & $0 \pm 0^{\mathrm{bc}}$ & $0 \pm 0^{\mathrm{bc}}$ & $0 \pm 0^{\mathrm{bc}}$ & $0 \pm 0^{\mathrm{bc}}$ \\
L16A1D9 & $0 \pm 0^{\mathrm{bc}}$ & $0 \pm 0^{\mathrm{bc}}$ & $0 \pm 0^{\mathrm{bc}}$ & $0 \pm 0^{\mathrm{bc}}$ & $0 \pm 0^{\mathrm{bc}}$ \\
L18A1D9 & $0 \pm 0^{\mathrm{bc}}$ & $3,24 \pm 4,58^{\mathrm{c}}$ & $4,9 \pm 6,36$ & $13,15 \pm 9,34^{\mathrm{ab}}$ & $15,8 \pm 14,3^{\mathrm{a}}$ \\
L22A1D9 & $0 \pm 0^{\mathrm{c}}$ & $0 \pm 0^{\mathrm{c}}$ & $0 \pm 0^{\mathrm{c}}$ & $0 \pm 0^{\mathrm{c}}$ & $0 \pm 0^{\mathrm{c}}$ \\
L5A1D9 & $0 \pm 0^{\mathrm{c}}$ & $0 \pm 0^{\mathrm{c}}$ & $0 \pm 0^{\mathrm{c}}$ & $0 \pm 0^{\mathrm{c}}$ & $0 \pm 0^{\mathrm{c}}$ \\
\hline
\end{tabular}

Nombre de nouvelles feuilles

\begin{tabular}{lccccc}
\hline L10A1D9 & $0 \pm 0^{\mathrm{b}}$ & $0 \pm 0^{\mathrm{b}}$ & $0 \pm 0^{\mathrm{b}}$ & $1,2 \pm 1,7^{\mathrm{b}}$ & $0 \pm 0^{\mathrm{b}}$ \\
L16A1D9 & $0 \pm 0^{\mathrm{b}}$ & $0,2 \pm 0,3^{\mathrm{b}}$ & $0 \pm 0^{\mathrm{b}}$ & $0,8 \pm 0,56^{\mathrm{b}}$ & $1,1 \pm 0,6^{\mathrm{b}}$ \\
L18A1D9 & $1,56 \pm 1,37^{\mathrm{b}}$ & $2,3 \pm 1,9^{\mathrm{ab}}$ & $1,8 \pm 1,37^{\mathrm{ab}}$ & $2,7 \pm 1,6^{\mathrm{ab}}$ & $3,9 \pm 1,6^{\mathrm{a}}$ \\
L22A1D9 & $0 \pm 0^{\mathrm{b}}$ & $0 \pm 0^{\mathrm{b}}$ & $0 \pm 0^{\mathrm{b}}$ & $0 \pm 0^{\mathrm{b}}$ & $0 \pm 0^{\mathrm{b}}$ \\
L5A1D9 & $0 \pm 0^{\mathrm{b}}$ & $0 \pm 0^{\mathrm{b}}$ & $0 \pm 0^{\mathrm{b}}$ & $0,3 \pm 0,47^{\mathrm{b}}$ & $0 \pm 0^{\mathrm{b}}$ \\
\hline
\end{tabular}

Matière fraîche totale $(g)$

\begin{tabular}{lccccc}
\hline L10A1D9 & $0 \pm 0^{\text {cd }}$ & $0 \pm 0^{\text {cd }}$ & $0 \pm 0^{\text {cd }}$ & $0,83 \pm 1,17^{\text {cd }}$ & $0 \pm 0^{\text {cd }}$ \\
L16A1D9 & $0 \pm 0^{\text {d }}$ & $1,05 \pm 1,06^{\text {cd }}$ & $0,6 \pm 0,5^{\text {cd }}$ & $1,44 \pm 1,02^{\text {bcd }}$ & $2,98 \pm 1,68^{\text {abc }}$ \\
L18A1D9 & $1,76 \pm 1,2^{\text {bcd }}$ & $1,9 \pm 1,38^{\text {bcd }}$ & $2,9 \pm 0,48^{\text {abc }}$ & $4,2 \pm 0,75^{\text {a }}$ & $3,58 \pm 1,99^{\text {ab }}$ \\
L22A1D9 & $0 \pm 0^{\text {d }}$ & $0 \pm 0^{\text {d }}$ & $0 \pm 0^{\text {d }}$ & $0 \pm 0^{\mathrm{d}}$ & $0 \pm 0^{\mathrm{d}}$ \\
L5A1D9 & $0 \pm 0^{\text {d }}$ & $0 \pm 0^{\text {d }}$ & $0 \pm 0^{\text {cd }}$ & $0,85 \pm 1,2^{\text {cd }}$ & $0 \pm 0^{\text {d }}$
\end{tabular}

\section{Matière sèche totale (g)}

\begin{tabular}{lccccc}
\hline L10A1D9 & $0 \pm 0^{\text {bcd }}$ & $0 \pm 0^{\text {bcd }}$ & $0 \pm 0^{\text {bcd }}$ & $0,41 \pm 0,58^{\text {bcd }}$ & $0 \pm 0^{\text {bcd }}$ \\
L16A1D9 & $0 \pm 0^{\text {bcd }}$ & $0,4 \pm 0,35^{\text {bcd }}$ & $0,36 \pm 0,32^{\text {bcd }}$ & $0,87 \pm 0,63^{\text {abcd }}$ & $1,18 \pm 0,56^{\text {bcd }}$ \\
L18A1D9 & $1,04 \pm 0,8^{\text {abcd }}$ & $0,71 \pm 0,5^{\text {bcd }}$ & $1,09 \pm 0,23^{\text {abc }}$ & $1,7 \pm 0,13^{\mathrm{a}}$ & $1,8 \pm 0,17^{\mathrm{a}}$ \\
L22A1D9 & $0 \pm 0^{\mathrm{d}}$ & $0 \pm 0^{\mathrm{cd}}$ & $0 \pm 0^{\mathrm{cd}}$ & $0 \pm 0^{\mathrm{cd}}$ & $0 \pm 0^{\mathrm{bcd}}$ \\
L5A1D9 & $0 \pm 0^{\mathrm{bcd}}$ & $0 \pm 0^{\mathrm{bcd}}$ & $0 \pm 0^{\mathrm{bcd}}$ & $0,38 \pm 0,53^{\mathrm{bcd}}$ & $0 \pm 0^{\mathrm{cd}}$
\end{tabular}

*Sur la même ligne, les données portant les mêmes lettres ne sont pas significativement différentes au seuil de 5\%. Test de Newman-Keuls. 


\section{DISCUSSION}

L'essai de bouturage sous tunnel du colatier en pépinière a abouti à des taux de mortalité importants des boutures. Le taux de survie moyen général des boutures est faible $(5,9 \%)$. Le meilleur taux de survie, $41,2 \%$, a été obtenu avec le génotype L18A1D9. Les résultats de l'étude montrent l'effet prépondérant du génotype et celui du nombre de feuilles laissées par bouture, au cours du bouturage sous tunnel. Ainsi l'effet significatif du génotype a laissé apparaître une différence de taux de survie moyen entre les génotypes de colatiers quel que soit le nombre de feuilles. Ces résultats viennent corroborer ceux de Koko et collaborateurs en 2011, qui avaient constaté dans les mêmes conditions, une grande influence de la plante mère, donc du génotype, dans la réussite du bouturage du cacaoyer plante de la même famille que le colatier. Ils viennent également renforcer les difficultés évoquées lors des premiers essais de bouturage de Cola nitida, effectués en Côte d'Ivoire. Ces essais ont mis en évidence, les faibles taux de réussite dû à l'effet du génotype et au faible niveau d'enracinement.

Concernant le nombre de feuilles adéquat par boutures, il est admis que la présence de feuilles sur les boutures a un effet sur l'initiation des racines, donc la survie des boutures. Il existerait alors une surface foliaire optimale capable d'induire un taux d'enracinement optimum pour une espèce donnée (Tchoundjeu et al., 2002 ; Atangana et al., 2006).Une surface foliaire optimale est celle qui permet un équilibre entre le gain en assimilations photosynthétiques et la perte d'eau par transpiration de la bouture (Tchoundjeu, 2004). Les meilleurs taux de survie $(41,2 \%)$, taux d'enracinement $(88,9 \%)$ et de croissance en longueur ont été obtenus avec le génotype L18A1D9 pour des boutures avec 4 feuilles. Les feuilles jouent un rôle important dans la survie des boutures. La migration des hydrates de carbone à partir des feuilles contribue à la formation des racines.

Aussi un nombre trop ou peu élevé de feuilles agît sur la transpiration et l'hydratation des boutures. Le facteur «Nombre de feuille » agit donc sur la reprise des boutures, la croissance et le développement de leur biomasse. D'où la nécessité de recommander le bouturage avec quatre (4) feuilles par bouture en ce qui concerne le colatier (Cola nitida) indépendamment $\mathrm{du}$ génotype. L'analyse détaillée des données de la biomasse fraîche et sèche (totale, aérienne, racinaire) ont confirmé l'effet prépondérant du génotype et de la surface foliaire. Elle a mis en évidence une bonne aptitude au bouturage du génotype L18A1D9 et les meilleurs taux de survie des boutures avec 4 feuilles.

En plus des deux facteurs étudiés dans cet essai et pour améliorer les taux de réussite, d'autres facteurs, notamment le substrat et la période de bouturage, devront être pris en compte dans les études ultérieures. En effet le substrat de culture joue un rôle important dans la réussite $\mathrm{du}$ bouturage en pépinière. L'utilisation du terreau en pépinière pour le bouturage a été maintes fois recommandée pour plusieurs espèces végétales (Allanblackia floribunda, Atangana et al., 2006 ; Baillonella toxisperma, Ngo Mpeck et Atangana, 2007 ; Cola acuminata, Paluku et al., 2018). C'est un substrat jugé approprié pour l'enracinement de boutures avec un volume optimal de pores, une bonne réserve en éléments nutritifs et un taux de diffusion d'oxygène adéquat pour la respiration des racines des boutures (Andersen, 1986 ; Kontoh, 2014). Cependant des études ont montré des préférences pour certaines espèces végétales, soit pour la sciure de bois, soit pour le mélange sciure de bois plus terreau (Tchoundjeu et al., 2004; Tsobeng et al., 2011).

A l'issus de cette étude, nous retenons que de nombreux facteurs influencent la réussite du bouturage sous tunnel du colatier. En plus des facteurs d'origines physiologiques telles que les différences génétiques entre les génotypes et la surface foliaire ; nous pouvons ajouter l'âge physiologique des rameaux et les facteurs nutritionnels (Kouamenan et al., 2001; Amoah et al., 2006b ; Koko et al., 2011). Les périodes de saisons sèches sont à éviter car elles favoriseraient une déshydratation plus importante des boutures malgré l'arrosage et accentueraient le taux de mortalité (Ricez, 2008). 


\section{Conclusion}

Cette étude a consisté à évaluer l'influence sur le bouturage du colatier de deux facteurs : le génotype du matériel végétal utilisé et le nombre de feuilles par bouture. L'étude menée à la pépinière du CNRA de Man a permis d'identifier le génotype L18A1D9 comme le génotype le plus apte au bouturage dans le cadre de notre étude $(41,7 \%)$. Il ressort également de cette étude qu'il faut laisser 4 feuilles par bouture lors du bouturage sous tunnel du colatier pour obtenir un taux de survie acceptable et un bon enracinement $(88,9 \%)$. Les travaux futurs pourront être orientés vers la recherche du meilleur substrat, de la meilleure période de bouturage et l'utilisation des auxines de synthèses pour l'amélioration de l'enracinement et du taux de survie des boutures.

\section{CONFLIT D'INTERETS}

Les auteurs déclarent n'avoir aucun conflit d'intérêts.

\section{CONTRIBUTIONS DES AUTEURS}

Ce travail a été fait au Centre National de Recherche Agronomique (Côte d'Ivoire) dans le cadre du projet "Amélioration de la productivité du colatier en Côte d'Ivoire » sous la supervision de BB et J-MDS. RG a conduit et collecté les données de l'expérience. YO et NG ont aidé à concevoir les protocoles. HL et JZK étaient Co superviseurs de ce travail en tant que collaborateurs sur ce projet. BB est le coordinateur du projet.

\section{REMERCIEMENTS}

Nous sommes reconnaissants au Fonds Interprofessionnel pour la Recherche et le Conseil Agricoles (FIRCA) qui a financé le projet «Amélioration de la productivité du colatier en Côte d'Ivoire " dans le cadre duquel cette étude a été réalisée.

\section{REFERENCES}

Adebola PO. 2003. Genetic characterization and biosystematics studies in the genus Cola Schott and Endlicher. PhD thesis, University of Ibadan, Nigeria, 203 P.
Adenuga OO, Mapayi EF, Olasupo FO, Olaniyi OO, Oyedokun AV. 2012. Nigeria's Cola genetic ressources: the need for renewed exploration. Asian $J$. Agric. Sci., 4: 177-182.

Aloko-N'guessan J. 2000. Cola, espace et sociétés étude de géographie sociale et culturelle de la filière de la noix cola au marché de Gros de Bouaké. Revue CAMES - Série B, (02): 11 p.

Amoah FM. 2006b. Review of vegetative propagation of cacao (Theobroma cacao L.) by rooted cuttings. Environmental and technical considerations. Ghana $J$. of Agric. Sci., 39: 217-226. DOI: http://dx.doi.org/10.4314/gjas.v39i2.214 5

Andersen AS. 1986. Stock plant conditions. In New Root Formation in Plant and Cuttings, Jackson MB (Ed). Martinus Njihoff: Dordrecht; 223-255.

Asogwa EU, Anikwe JC, Mokwunye FC. 2006. Kola production and utilization for economic development. African Scientist., 7(4): 217-222.

Atangana AR, Tchoundjeu Z, Asaah EK, Simons AJ, Khasa DP. 2006. Domestication of Allanblackia floribunda: Amenability to vegetative propagation. Forest Ecol. and Manag., 237: 246-251. DOI: http://dx.doi:10.1016/j.foreco.2006.09.08 1

Berté K. 2009. Etat des lieux de la filière cola en Côte d'Ivoire. Rapport FIRCA, 87 p.

Biégo HM, Yao DK, Ezoua P, Kouame CO et Kouadio LP. 2009. Niveaux de contamination en pesticides organochlorés des noix de Cola nitida. Int. J. Biol. Chem. Sci., 3(6): 1238-1245.

Calvet G, Villemin P. 1986. Interprétation des analyses de terre. SCPA, France, 24 p.

Degrande A, Facheux C. 2002. Production et Commercialisation des plantes produites dans les pépinières villageoises: Guide pratique pour formateurs, encadreurs et pépiniéristes. ICRAF West Africa, World Agroforestry Centre, $52 \mathrm{p}$.

Gbédié NA, Bonsson B, Ouattara Y, Bahan LMF, Kouadio KT, Traoré SM, Légnate NH, Keli ZJ, 2017. Méthodes de levée 
de dormance de la noix de cola fraîche (Cola nitida [Vent.] Schott et Endlicher). J. Appl. Biosci., 120: 11999-12005. DOI: https://dx.doi.org/10.4314/jab.v120i1.2

INRA. 1995. Référentiel pédologique. Association Française d'étude des sols. INRA.

Koko L, Koffi N, Konan A. 2011. Multiplication végétative du cacaoyer (Theobroma cacao L.) par la technique de bouturage direct sous tunnel plastique. J. Appl. Biosci., 46: 31243132.

Kouamanan OK. 2001. Multiplication végétative du cacaoyer: le bouturage. Mémoire de Maîtrise, Université d'Abobo-Adjamé, Côte d'Ivoire, p.40.

Mbete P, Makosso S, Lelou B, Douh C, Ngokaka C. 2011. Essai de multiplication végétative du Colatier (Cola nitida) via la technique du marcottage au Congo Brazzaville. JABs., $37: 2485-2490$.

MINADER (Ministère de l'Agriculture et du Développement Rural). 2018. http://www.agriculture.gouv.ci/. (25/08/2019).

Ngo-Mpeck ML, Atangana A. 2007. Rooting of Leafy Stem Cuttings of Baillonella toxisperma. J. For. Sci., 53(5): 571-579.

Odutayo OI, Adeyemi FA, Adebola PO, Sotimehim OI, 2018. Compatibility studies in Cola nitida genotypes. JPBCS., 10(4): 80-85. DOI: https://dx.doi.org/10.5897/JPBCS2017.0 637

Ouattara Y, Bonsson B, Traore MS, Gbédié NA, Akaffou DS, Sie RS, Keli ZJ. 2018 Évaluation de la diversité agromorphologique d'accessions de colatiers (Cola nitida (Vent.) Schott et Endlicher) collectées au Sud et au Sud-Ouest de la Côte d'Ivoire. J. Appl. Biosc., 122: 12296-12308.

DOI: http://dx.doi.org/10.4314/jab.v122i1.9

Oladokun MAO. 2000. Kola: Tree of life. Thesis, University of Agriculture, Abeokuta. p.37.
Paluku A, Okungo A Bwama M. 2018a. Bouturage de Cola acuminata (P. Beauv.) Schott and Endl.: Influence du substrat, de la longueur et de la surface foliaire sur l'enracinement de boutures à Kisangani, RD Congo. JABs., 123:12354-12362. DOI: https://dx.doi.org/10.4314/jab.v123i1.4

Paluku A, Bwama M, Okungo A, Van damme P. 2018b. Multiplication végétative de Cola acuminata (Pal. de Beauv.) Schott \& Endlicher par marcottage à Kisangani, République Démocratique du Congo. Int. J. Biol. Chem. Sci., 12(3): 1141-1150. DOI:

http://dx.doi.org/10.4314/ijbcs.v12i3.6

Tchoundjeu Z, Kengeu J. and Leakey RRB. 2002. Domestication of Dacryodes edulis: State-of-the Art. For. Trees Livelihoods, 12: 3-13.

Tchoundjeu Z, Ngo Mpeck ML, Asaah E, Amougou A. 2004. The role of vegetative propagation in the domestication of Pausinystalia johimbe (K. Schum), a highly threatened medicinal species of West and Central Africa. Forest Ecol. Manag. J., 188 (1): 175-183.

Ricez T. 2008. Etude des modes de régénération à faible Coût de Prosopis africana et Detarium microcarpum en forêt classée de Dinderesso. Master II 'Bioressources en régions tropicales et méditerranéennes', Université Paris XII, p.60.

Tsobeng A, Tchoundjeu Z, Kouodiekong L, Asaah E. 2011. Effective propagation of Diospyros crassiflora (Hiern) using twing cuttings. Int. J. Biosci., 4: 109117.

Whitlock BA, Bayer C, Baum DA. 2001. Phylogenetic relationships and floral evolution of the Byttnerioideae (Sterculiaceae or Malvaceae s.1.) based on sequences of the chloroplast gene, ndhF. Syst. Bot., 26(2) : 420-437. 